$v_{z}$ component; this force alone would give $\dot{v}_{\varepsilon}=\frac{\mu}{m} \frac{\partial \varphi}{\partial z}$. On account of the relation $\dot{\beta \beta}=$ $-\frac{v_{s} v_{s}}{v^{2}}$, this adds a term $-\frac{\mu}{m v^{2}} \frac{\partial \varphi}{\partial z} \frac{v_{z}}{\beta}$ to the variation of $\beta$; so we must write

$$
\frac{d \beta}{d t}=\frac{\beta_{0}^{2}}{2 \beta \varphi_{0}} \frac{\partial \varphi}{\partial z} v_{z}-\frac{\mu}{m v^{2}} \frac{\partial \varphi}{\partial z} \frac{v_{z}}{\beta} \text { or } \frac{d \beta}{d z}=\frac{1}{v_{z}} \frac{d \beta}{d t}=\frac{1}{\beta} \frac{\partial \varphi}{\partial z}\left[\frac{\beta_{0}^{2}}{2 \varphi_{0}}-\frac{\mu}{m v^{2}}\right] .
$$

The integration is immediate and yields $\beta^{2}=\beta_{0}^{2} \frac{\varphi}{\varphi_{0}}-\frac{2 \mu}{m v^{2}}\left(\varphi-\varphi_{0}\right)$. The stopping field is obtained for $\beta=1$, and, on account of the fact that the term in $\mu$ is very small, we can write

$$
\varphi_{1}=\frac{\varphi_{0}}{\beta_{0}^{2}}\left[1+\frac{2 \mu}{m v^{2}}\left(\varphi-\varphi_{0}\right)\right]=\varphi\left[1+\frac{2 \mu}{m v^{2}}\left(\varphi-\varphi_{0}\right)\right] .
$$

In the integration, we have treated $m v^{2}$ as a constant, an approximation actually justified if we take account of the very small magnitude of the magnetic forces.

\title{
ON THE DISTRIBUTION LAW IN LOCALLY RAPIDLY FLUC- TUATING FIELDS WHICH ARE STEADY WHEN AVERAGED OVER A SUFFICIENT TIME INTERVAL
}

\section{By Benedict CASSEN}

Norman Bridge laboratory of Physics, California Institute of Themology

\section{Communicated August 27, 1928}

Thomas $^{1}$ and later Fermi ${ }^{2}$ have attempted to determine the time average electrical potential around the nucleus of a heavy atom. They treat the electrons as if they formed an ideal degenerate Fermi gas which becomes statistically distributed in the steady field due to the nucleus plus the locally rapidly fluctuating field due to the electrons themselves. This method is apparently fully justified and it is hard to see, at first, why the numerical solutions given by the above writers turn out to be so roughly approximate, especially for heavy atoms with many electrons. The following investigation purports to show that the roughness of the method is due to the unjustified, except for approximation purposes, use of the statistical distribution law of an ideal gas in a steady field in the case where the field is steady only when averaged over a sufficiently long time interval and is locally fluctuating. It is shown that if there is any correlation between the local fluctuations in density and local fluctuations in the field that additional time-average forces arise which are derivable from a potential. This "correlation potential" must be introduced in the distribution law in order that the above-mentioned method should be precise. 
Consider a small element of volume $\Delta v$ in a locally fluctuating field of force, which is so small that the fraction of the time that more than one particle of an ideal gas acted upon by this field is in $\Delta v$ is negligible. Let $G_{k}(t)(k=1,2,3)$ be the component of the instantaneous forces per unit mass acting on $\Delta v$. Let $\rho(t)$ be the instantaneous density in $\Delta v$. $\rho(t)$ is on the whole equal to $m / \Delta v$ or 0 , where $m$ is the mass of a particle. Then the instantaneous component of force on the element $\Delta v$ is $G_{k}(t)$ $\rho(t) \Delta v$.

Let $T$ be a sufficiently long time interval so that the distribution of the gas and the field are steady when averaged over the time $T$. The average forces on gas in $\Delta v$ are then $(1 / T) \mathscr{C}^{T} G_{k}(t) \rho(t) \Delta v d t$. For time average momentum equilibrium in the element $\Delta v$ we have

$$
\frac{\partial p}{\partial x_{k}} \Delta v=\frac{1}{T} \int_{0}^{T} G_{k}(t) \rho(t) \Delta v d t
$$

where $p$ is the pressure which is already a time-averaged quantity.

Let $g_{k}=(1 / T) \int_{0}^{T} G_{k}(t) d t$ be the average value of $G_{k}(t)$ and let $r=$ $(1 / T) \int_{0}^{T} \rho(t) d t$ be the average value of $\rho(t)$. Let $h_{k}(t)=G_{k}(t) g-g_{k}$ and $s(t)=\rho(t)-r$ be the corresponding instantaneous deviations from the average values. Then (1) becomes

that is,

$$
\frac{\partial p}{\partial x_{k}}=\frac{1}{T} \int_{0}^{T}\left[h_{k}(t) s(t)+h_{k}(t) r+g_{k} s(t)+g_{k} r\right] d t,
$$

$$
\frac{\partial p}{\partial x_{k}}=g_{k} r+\frac{1}{T} \int_{0}^{T} h_{k}(t) s(t) d t .
$$

The second term on the right will only vanish when there is no correlation between the deviations from the average of density and the deviations from average of the external force per unit mass. When it does vanish then

$$
\frac{\partial p}{\partial x_{k}}=g_{k} r
$$

If the temperature is uniform the law of statistics enables one to determine $p$ as a function $p(r)$ of $r$ so that, as in the classical distribution law

$$
g_{k}=\frac{\partial}{\partial x_{k}} \int \frac{d p}{r}=-\frac{\partial V}{\partial x_{k}},(k=1,2,3)
$$

which gives

$$
V=-\int \frac{d p}{r}=\text { const. }
$$

which on evaluating the integral and solving for $r$ gives the distribution law sought for. 
In the case where the correlation term in (2) does not vanish, which it does not in general, we have, assuming the time-average forces per unit mass to be derivable from a time-average potential $V$,

$$
\frac{\partial}{\partial x_{k}} \int \frac{d p}{r}+\frac{\partial V}{\partial x_{k}}=\frac{1}{r T} \int_{0}^{T} h_{k}(t) s(t) d t,(k=1,2,3) .
$$

The right-hand side is evidently derivable from a potential

$$
R=\int \frac{d p}{r}+V+c
$$

where $c$ is an integration constant. Let us call $R$ the correlation potential.

For example, for the case of Maxwellian statistics $p=r k T / m$ so that

$$
R=\frac{k T}{m} \log r+V+c .
$$

If $R$ is, in any case, independent of $r$ then

$$
r=r_{0} e^{m(R-V) / k T}
$$

and if $R$ vanishes the law becomes the classical one.

In the case of an almost degenerate Fermi gas we have, when the weight factor $=2$,

$$
p=\frac{8 \pi h^{2}}{15 m}\left(\frac{3 r}{8 \pi m}\right)^{0 / 3}+\frac{2 m \pi}{9 h^{2}}\left(\frac{3 r}{8 \pi m}\right)^{1 / 3}(2 \pi k T)^{2}
$$

so that

$$
R=\frac{4 \pi h^{2}}{3 m}\left(\frac{3}{8 \pi m}\right)^{5 / 3} r^{2 / 3}-\frac{m \pi}{9 h^{2}}\left(\frac{3}{8 \pi m}\right)^{1 / 3}(2 \pi k T)^{2} r^{-2 / 3}+V+c
$$

or solving for $r$ if $R$ is independent of $r$

$$
r=\frac{8 \pi m^{4}}{3 h^{3}}\left[(R-V-c)+\sqrt{(R-V-c)^{2}+\frac{1}{3}\left(\frac{\pi k T}{2 m}\right)^{2}}\right]^{3 / 2}
$$

For a completely degenerate gas this becomes

$$
r=\frac{8 \pi m^{4}}{3 h^{3}} \cdot 2^{3 / 2}(R-V-c)^{3 / 2}
$$

and if $R$ vanishes we get the law used by Thomas and Fermi.

The unmodified form of the distribution law for the case of Maxwellian statistics is used in the well-known Debye-Hückel theory of electrolytic solutions. The theory is also roughly approximate except when applied to extremely dilute solutions. Changes in the results by corrections made in order to take care of the analytical approximations do not lead to com- 
pletely satisfactory results. In concentrated solutions of electrolytes, when the ions are crowded together, correlation forces arising from systematic interaction of the ions are to be expected to occur, and then the fundamental equation obtained by eliminating the time-average charge density between a time-average Poisson's equation and the distribution law will be modified by a correlation potential. The no doubt important alterations due to this modification will have to be taken into account in any satisfactory extension of the Debye theory to concentrated solutions. Pauling ${ }^{3}$ has shown that by introducing an extra potential in a semi-empirical way it is possible to get results giving the major features of the behavior of electrolytic solutions up to rather high concentrations. The present work indicates the probable physical nature of the effects requiring deviations from the simple Debye treatment and gives a physical interpretation to Pauling's method of correction.

1 Thomas, Proc. Camb. Phil. Soc., 23, 542-8 (1926).

2 Fermi, Z. Physik, 48, 73-9 (1928).

${ }^{3}$ Pauling, paper read at Los Angeles meeting of the American Chemical Society, June, 1925.

\section{THE CRYSTAL STRUCTURE OF POTASSIUM SULPHATE}

\section{By Frank Peat Goeder}

\section{Ryerson Physical laboratory, The University of Chicago}

Communicated September 5, 1928

In a previous paper ${ }^{1}$ the space group of potassium sulphate was determined from data obtained from Laue photographs to be $2 D i-13$. While from the point of view of descriptive crystallography this result is of fundamental importance, for crystal structure determinations it is of value only if it can lead to a solution of the complete internal structure of this substance. This paper is a brief account of the way in which the information obtained from the geometrical theory of space groups has resulted in a complete solution not only of the crystal structure of potassium sulphate but also presents for the first time a quantitative three-dimensional structure for the potassium sulphate molecule. Furthermore, the results of $\mathrm{x}$-ray powder photographs of potassium sulphate are presented, and the diffraction effects computed from the structure obtained from the space group are shown to be quantitatively verified in every detail by experiment.

As was previously shown, ${ }^{1}$ the elementary lattice of potassium sulphate contains four molecules of $\mathrm{K}_{2} \mathrm{SO}_{4}$ and has the lattice constants $a_{0}=5.746$ A.U., $b_{0}=10.033$ A.U. and $c_{0}=7.443$ A.U., as determined by Ogg and Hopwood. The author of this paper redetermined the lattice constants 